\section{The behavioral/cognitive status of motor neuron disease patients should be assessed with disease-specific tools}

\section{Stefano F Cappa}

Division of Neuroscience, Vita-Salute University \& San Raffaele Scientific Institute, Milan, Italy - cappa.stefano@hsr.it

Evaluation of: Raaphorst J, Beeldman E, Schmand B et al. The ALS-FTD-Q: a new screening tool for behavioral disturbances in ALS. Neurology 79(13), 1377-1383 (2012). The issue of cognitive and behavioral disorders in motor neuron disease is the focus of intensive investigation. The screening tool developed by Raaphorst et al. represents a clinically significant advancement, because of its excellent psychometric properties. Further studies are needed to assess the relationship between behavioral disturbances, executive dysfunction and social cognition impairment in motor neuron disease.

The concept of motor neuron disease (MND) as a disorder that 'spares the intellect' has gone relatively unchallenged for many years. Two kinds of observation opened the discussion. First, clinical case studies were published, reporting the association of MND with dementia [1]. While in the early reports the clinical features of dementia were not described in detail, in 1990 Neary and colleagues [2], reported four cases in a landmark paper in which the pattern of neuropsychological impairment, the single PET imaging data and the pathological findings "resembled those of dementia of frontal-lobe type and were distinct from those of Alzheimer's disease." The second line of investigation dealt with neuropsychological studies of nondemented MND patients, and supported the presence of subclinical impairment, with relative sparing of memory function [3]. Subsequent studies have, in general, documented an impairment of executive function [4]. More recently, the identification of TDP-43 as a constituent of ubiquitin-positive inclusions in both frontotemporal dementia and sporadic amyotrophic lateral sclerosis (ALS), as well as the recent observations that mutations in C9ORF can be associated with both conditions, have provided further support for the concept of an overlap between these two entities [5].

Some screening tests have been proposed for the cognitive and behavioral assessment of MND patients. These include the Penn State Screening Battery, which is based on the assessment of verbal associative fluency, verbal abstract reasoning and judgment [6], emphasizing the cognitive, rather than the behavioral, disturbances, and the ALS Cognitive Behavioral Screen, which combines a brief cognitive assessment with a 15 -item behavioral questionnaire for caregivers [7].

The reasons why the evaluation of MND patients needs to include a comprehensive behavioral and cognitive assessment are multiple. At the moment, these include factors such as prognostication of clinical course (survival appears to be negatively correlated with cognitive/behavioral dysfunction [8]), support to the family/caregivers [9] and adherence to therapeutic interventions and management decisions. One hopes to be able to address, in the future, crucial issues such as a more precise definition of the phenotypic heterogeneity of the disease spectrum and response to disease-modifying therapies.

\section{Methods \& results}

The development of disease-specific, comprehensive rating scales for the assessment of behavioral manifestations in MND is extremely important for clinical practice and research purposes, and the contribution of Raaphorst and colleagues represents a significant advancement in this area [10]. The main strength of the behavioral questionnaire lies in the selection of the items, which is based on a careful analysis of the pertinent literature, rather than on a priori definition of expected consequences of frontal lobe involvement. This empirically driven, relatively theory-independent approach is particularly useful in the domain of frontal lobe function, in which many different models have been proposed over the years, sometimes on the basis of limited or biased information. One additional remarkable methodological feature is
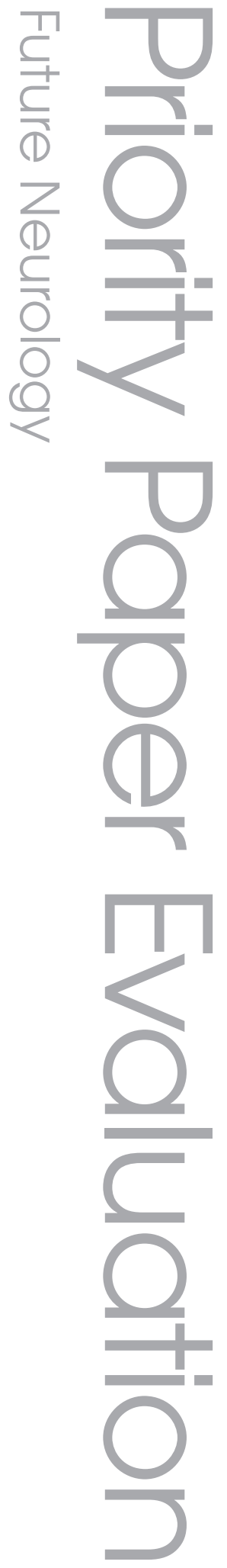

\section{Keywords}

- behavioral scale = cognition

- frontotemporal dementia

- motor neuron disease

Future Medicine part of 
the adoption of positive (both with and without ALS) and negative (muscle disease and general neurological) control groups.

The psychometric properties of the tool have been carefully evaluated, and appear to be quite satisfactory, both in terms of reliability and validity. It is to be underlined that the correlation is high with other behavioral scales, supporting the construct validity of the scale, but only moderate with cognitive/executive test scores, providing further evidence for the possible dissociation between these two facets of frontal lobe dysfunction. It is important to support the introduction of this 'user-friendly' questionnaire into widespread clinical usage, because the collection of additional data in large populations is required to assess the specificity and sensitivity of the measure, and to evaluate its possible usefulness in the detection of changes in the follow-up of individual patients.

\section{Discussion \& significance}

A careful assessment of cognition and behavior is mandatory in MND patients. Consensus criteria have been proposed by Strong and colleagues [11]. A diagnosis of cognitive impairment occurs with cognitive performance at or below the fifth percentile on at least two standardized neuropsychological tests sensitive to executive functioning. The impairment must not be accounted for by the patient's premorbid intellectual level, depression, bulbar dysfunction (dysarthria) or motor weakness. The diagnosis of behavioral impairment requires that individuals meet at least two nonoverlapping supportive diagnostic features from the Neary criteria [12]. The consensus paper provides a detailed list of neuropsychological tests which can be applied to the evaluation of executive function.

For the assessment of behavioral impairment, the suggested questionnaires are the Frontal Behavioral Inventory [13], the Neuropsychiatric Inventory [14], the Frontal Systems Behavior Scale [15] and the Cambridge Behavioral Inventory Revised [16]. However, these instruments have not been specifically developed for MND patients, and they include items which can only be evaluated in the absence of relevant motor dysfunction. The ALS-Frontotemporal Dementia Questionnaire represents an important addition to the available instruments, and is likely to be used extensively for clinical and research purposes.

\section{Conclusion \& future perspective}

Future developments in the area of extra-motor dysfunction in MND need an updated conceptual framework. The traditional idea that associates executive dysfunction with involvement of the frontal convexity, and behavioral disturbances with orbitofrontal damage clearly represents a gross oversimplification of the complex relationship between the frontal lobe and behavior. In recent years, the (still crucial) insights provided by the study of patients with frontal damage have been extensively integrated by research into frontal lobe functions in nonhuman primates and by neuroimaging investigations of normal human subjects. The recent increasing interest in the neural mechanisms of social cognition is largely the result of these combined research efforts. Consider, for example, empathy, a complex and heterogeneous construct, the definition and functional characterization of

\section{Executive summary}

\section{Background}

- Cognitive and behavioral impairment can be observed in patients with a diagnosis of motor neuron disease (MND).

- Most of the available assessment tools have not been developed specifically for MND patients.

\section{Methods \& results}

- A specific assessment instrument, the Amyotrophic Lateral Sclerosis-Frontotemporal Dementia Questionnaire, was developed on the basis of a literature evaluation of the cognitive and behavioral features of MND.

- A comprehensive psychometric and clinimetric evaluation confirmed the reliability and validity of the questionnaire.

\section{Discussion \& significance}

- It is now mandatory to assess cognitive and behavioral status in MND patients.

- The availability of a short, disease-specific instrument will be useful for clinical and research purposes.

\section{Conclusion \& future perspective}

- In-depth assessment of social cognition abilities in MND patients may shed new light on the pathogenesis of cognitive and behavioral symptoms.

- In vivo correlation with measures of extra-motor brain involvement, as well as genetic analysis, are required to support the hypothesis of a fronto-temporal-to-MND pathological spectrum. 
which is still debated [17]. Empathic abilities cover several phenomena, ranging from automatic affect sharing (i.e., emotional contagion) to the attribution of mental states, including emotional states or intentions. At the neural level, representing different types of another individual's mental states engages partially overlapping and partially segregated neural systems. Imaging studies in normal subjects have associated a general ability in the attribution of mental states (i.e., mentalizing) with a broad network of areas, including the medial prefrontal cortex, the temporo-parietal junction and the temporal poles. By contrast, the attribution of emotional states appears to involve the fronto-insular cortex and the amygdala. Both aspects of mentalizing have been found to be affected in patients with MND $[18,19]$. The possible involvement of specific cerebral regions in MND patients can be assessed with quantitative neuroimaging, using MRI or PET, and correlated with the results of social cognition tests. The relationship between executive dysfunction, behavioral disturbance and social cognition impairment can then be assessed at the individual subject level.

\section{Financial \& competing interests disclosure \\ The author has no relevant affiliations or financial involvement with any organization or entity with a financial interest in or financial conflict with the sub- ject matter or materials discussed in the manuscript. This includes employment, consultancies, honoraria, stock ownership or options, expert testimony, grants or patents received or pending, or royalties. \\ No writing assistance was utilized in the production of this manuscript.}

\section{References}

1. Hudson AJ. Amyotrophic lateral sclerosis and its association with dementia, parkinsonism and other neurological disorders: a review. Brain 104(2), 217-247 (1981).

2. Neary D, Snowden JS, Mann DM, Northen B, Goulding PJ, Macdermott N. Frontal lobe dementia and motor neuron disease. J. Neurol. Neurosurg. Psychiatry 53(1), 23-32 (1990).

3. Gallassi R, Montagna P, Ciardulli C, Lorusso S, Mussuto V, Stracciari A. Cognitive impairment in motor neuron disease. Acta Neurol. Scand. 71(6), 480-484 (1985).

4. Neary D, Snowden JS, Mann DM. Cognitive change in motor neurone disease/amyotrophic lateral sclerosis (MND/ALS). J. Neurol. Sci. 180(1-2), 15-20 (2000).

5. Majounie E, Renton AE, Mok Ket al. Frequency of the C9orf72 hexanucleotide repeat expansion in patients with amyotrophic lateral sclerosis and frontotemporal dementia: a cross-sectional study. Lancet Neurol. 11(4), 323-330 (2012).

6. Flaherty-Craig C, Eslinger P, Stephens B, Simmons Z. A rapid screening battery to identify frontal dysfunction in patients with ALS. Neurology 67(11), 2070-2072 (2006).
7. Grossman AB, Woolley-Levine $S$, Bradley WG, Miller RG. Detecting neurobehavioral changes in amyotrophic lateral sclerosis. Amyotroph. Lateral Scler. 8(1), 56-61 (2007).

8. Elamin M, Phukan J, Bede P et al. Executive dysfunction is a negative prognostic indicator in patients with ALS without dementia. Neurology 76(14), 1263-1269 (2011).

9. Chio A, Vignola A, Mastro E et al. Neurobehavioral symptoms in ALS are negatively related to caregivers' burden and quality of life. Eur. J. Neurol. 17(10), 1298-1303 (2010).

10. Raaphorst J, Beeldman E, Schmand B et al. The ALS-FTD-Q: a new screening tool for behavioral disturbances in ALS. Neurology 79(13), 1377-1383 (2012).

11. Strong MJ, Grace GM, Freedman M et al. Consensus criteria for the diagnosis of frontotemporal cognitive and behavioural syndromes in amyotrophic lateral sclerosis. Amyotroph. Lateral Scler. 10(3), 131-146 (2009).

12. Neary D, Snowden JS, Gustafson L et al. Frontotemporal lobar degeneration. A consensus on clinical diagnostic criteria. Neurology 51, 1546-1554 (1998).
13. Kertesz A, Davidson W, Fox H. Frontal behavioral inventory: diagnostic criteria for frontal lobe dementia. Can. J. Neurol. Sci. 24, 29-36 (1997).

14. Cummings JL, Mega M, Gray K, Rosemberg-Thompson S, Carusi DA, Gornbei J. The Neuropsychiatric Inventory: comprehensive assessment of psychopathology in dementia. Neurology 44, 2308-2314 (1994).

15. Stout JC, Ready RE, Grace J, Malloy PF, Paulsen JS. Factor analysis of the frontal systems behavior scale (FrSBe). Assessment 10(1), 79-85 (2003).

16. Wedderburn C, Wear H, Brown J et al. The utility of the Cambridge Behavioural Inventory in neurodegenerative disease. J. Neurol. Neurosurg. Psychiatry 79(5), 500-503 (2008).

17. Shamay-Tsoory SG. The neural bases for empathy. Neuroscientist 17, 18-24 (2011).

18. Gibbons ZC, Snowden JS, Thompson JC, Happé F, Richardson A, Neary D. Inferring thought and action in motor neurone disease. Neuropsychologia 45, 1196-1207 (2007).

19. Girardi A, Macpherson SE, Abrahams S. Deficits in emotional and social cognition in amyotrophic lateral sclerosis. Neuropsychology 25, 53-65 (2011). 